NOUVELLE

\section{Drogues addictives, protéine phosphatases et voie ERK}

Emmanuel Valjent, Denis Hervé, Jean-Antoine Girault
Laboratoire de Transduction

du signal et plasticité dans

le système nerveux, Inserm

et Université Pierre et Marie

Curie, U.536; Institut du Fer

à Moulin, 17, rue du Fer à

Moulin, 75005 Paris, France.

girault@

$\underline{\text { fer-a-moulin.inserm. fr }}$

des récepteurs Dl de la dopamine [3]. Les études par immunofluorescence ont permis de caractériser les neurones dans lesquels ERK est activée après injection d'amphétamine. L'augmentation de la forme phosphorylée de ERK n'est observée que dans une fraction des neurones épineux de taille moyenne qui expriment le récepteur $D 1$, suggérant l'existence d'un deuxième signal requis pour l'activation de cette voie [8]. Ce deuxième signal semble être la stimulation des récepteurs de type NMDA ( $\mathrm{N}$-méthyl-Daspartate) du glutamate $[6,8]$. Ainsi, la phosphorylation de ERK dans les neurones épineux du striatum se comporterait comme un détecteur de coïncidence, nécessitant la stimulation concomitante des récepteurs $D l$ de la dopamine et NMDA du glutamate [8].

Dans un travail récent, nous avons clarifié le mécanisme par lequel la dopamine contrôle l'activation de ERK dans les neurones épineux du striatum en réponse aux drogues toxicomanogènes [8]. Ce mécanisme implique l'inhibition de la protéine phosphatase 1 ( $\mathrm{PPl}$ ) par une protéine clé de la signalisation dopaminergique dans le striatum, la DARPP-32 (dopamine- and cAMP-regulated phosphoprotein, Mr 32,000) [8, 9]. Lorsqu'elle est phosphorylée sur la thréonine 34 par la protéine kinase activée par l'AMP cyclique, en réponse à la stimulation des récepteurs $D 1$, la DARPP-32 devient un puissant inhibiteur de PP1 [9]. La phosphorylation de ERK induite par les drogues toxicomanogènes est abolie dans le striatum de souris mutantes 
dépourvues de DARPP-32 ou chez des souris knock-in ayant une mutation ponctuelle de la Thr-34 de la DARPP-32 en alanine [8]. L'inhibition de PPl par la DARPP-32 est cruciale à deux niveaux: en empêchant la déphosphorylation de ERK par la tyrosine phosphatase STEP (striatal-enriched tyrosine phosphatase) et en augmentant la phosphorylation de MEK (MAPK/ERK kinase) en agissant directement sur cette kinase ou en amont (Figure 1).

De façon remarquable, ces voies de signalisation sont nécessaires à la mise en place des effets comportementaux durables des drogues addictives sans intervenir dans leurs effets immédiats. $\varepsilon$ n effet, le blocage pharmacologique de ERK, ainsi que la délétion de la DARPP-32 ou sa mutation Thr-34-Ala affectent peu les effets immédiats de la cocaïne ou de l'amphétamine sur l'activité des souris. $\varepsilon$ n revanche, dans ces situations expérimentales, certaines réponses retardées (sensibilisation) ne se développent pas à la suite d'une exposition unique ou répétée à ces drogues [8]. De plus, certaines réponses durables conditionnées par la drogue (préférence de place conditionnée) ne sont plus observées si la voie ERK est bloquée pharmacologiquement avant chaque séance de conditionnement [6]. Ces résultats montrent que l'activation de $\varepsilon R K$ dans le striatum a les propriétés d'un détecteur de coïncidence d'activation des récepteurs NMDA du glutamate et DI de la dopamine. L'effet des récepteurs DI s'explique par l'inhibition par la DARPP-32 de la PPl qui régule négativement la voie ERK à plusieurs niveaux. L'activation de ERK semble cruciale pour la mise en place des effets comportementaux durables des drogues d'abus, suggérant un mécanisme par lequel la dopamine pourrait participer à l'apprentissage facilité par la récompense. $\diamond$

Drugs of abuse, protein phosphatases, and ERK pathway

\section{RéFÉRENCES}

1. Schultz W. Getting formal with dopamine and reward. Neuron 2002; 36: 241-63.

2. Di Chiara G, Imperato A. Drugs abused by humans preferentially increase synaptic dopamine concentrations in the mesolimbic system of freely moving rats. Proc Natl Acad Sci USA 1988; 85: $5274-8$.

3. Berke JD, Hyman SE. Addiction, dopamine, and the molecular mechanisms of memory. Neuron 2000; $25:$ 515-32.

4. Kelley AE. Memory and addiction: shared neural circuitry and molecular mechanisms. Neuron 2004; 44 : 161-79.

5. Thomas GM, Huganir RL. MAPK cascade signalling and synaptic plasticity. Nat Rev Neurosci 2004; 5: 173-83.
6. Valjent $\varepsilon$, Corvol JC, Pages C, et al. Involvement of the extracellular signal-regulated kinase cascade for cocaine-rewarding properties. J Neurosci 2000; 20: 8701-9.

7. Valjent $\varepsilon$, Pages C, Herve D, et al. Addictive and non-addictive drugs induce distinct and specific patterns of ERK activation in mouse brain. Eur J Neurosci 2004; 19: 1826-36.

8. Valjent $\varepsilon$, Pascoli V, Svenningsson P, et al. Regulation of a protein phosphatase cascade allows convergent dopamine and glutamate signals to activate ERK in the striatum. Proc Natl Acad Sci USA 2005; 102: 491-6.

9. Svenningsson P, Nishi A, Fisone G, et al. DARPP-32: an integrator of neurotransmission. Annu Rev Pharmacol Toxicol 2004; $44: 269-96$.

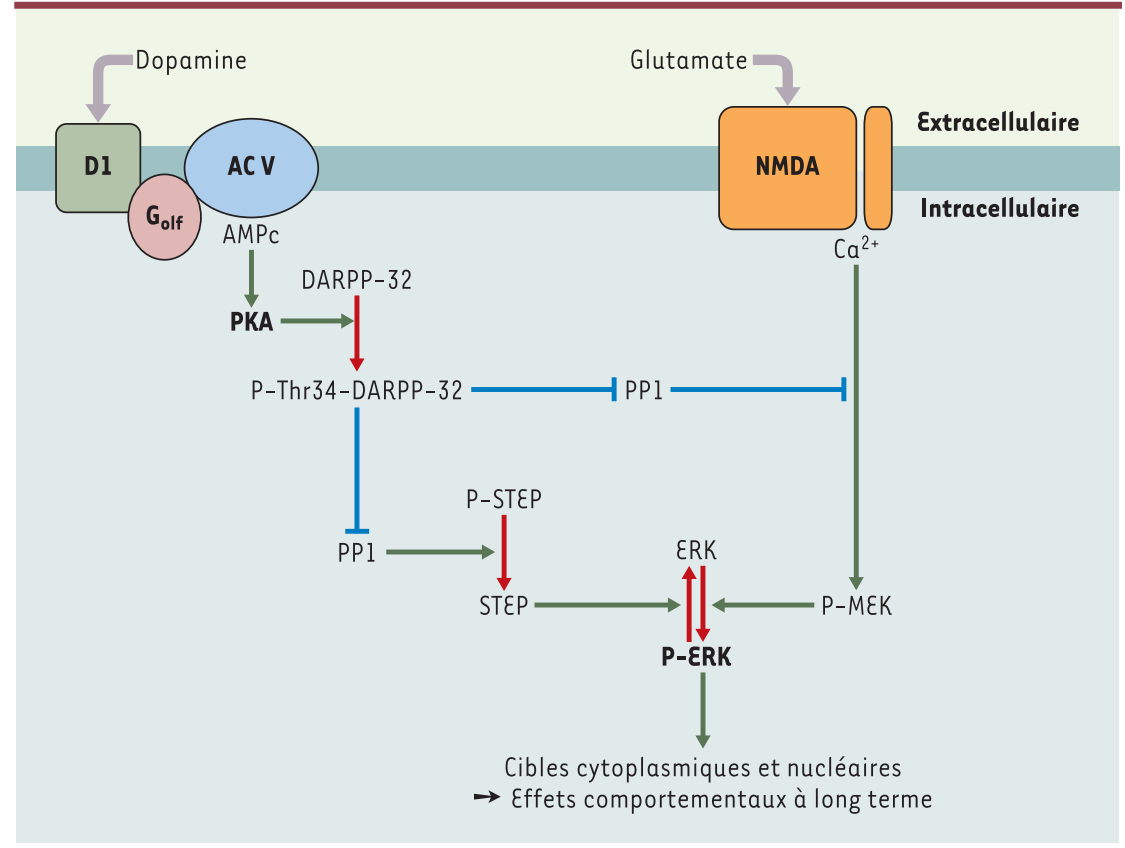

Figure 1. Activation de ERK dans les neurones du striatum. L'activation de ERK (extracellular signal regulated kinase) résulte de l'action concomitante du glutamate et de la dopamine [8]. Les récepteurs Dl de la dopamine activent l'adénylyl cyclase de type $V(A C V)$ par l'intermédiaire d'une protéine $G$ hétérotrimérique comprenant la sous-unité $\alpha_{\text {olf }}\left(G_{\text {olf }}\right)$. La protéine kinase activée par l'AMP cyclique (PKA) phosphoryle des cibles multiples parmi lesquelles la Thr-34 de la dopamine-and cAMP-regulated phosphoprotein Mr 32,000 (DARPP-32) qui devient ainsi un inhibiteur de la protéine phosphatase l (PPI). L'inhibition de la PPI a deux effets: elle favorise la phosphorylation de ERK en maintenant la tyrosine phosphatase STEP (striatal enriched phosphatase) sous forme phosphorylée inactive, et elle augmente l'activation de MEK (MAP-kinase/ERKkinase) en agissant directement sur celle-ci ou en amont. Cependant, la DARPP-32 phosphorylée ne pourrait à elle seule induire l'activation de ERK, celle-ci requiert par ailleurs une stimulation des récepteurs NMDA du glutamate ( $\rightarrow$ indique une action inhibitrice). 